\title{
Proteomic analysis of changes in protein expression in serum from animals exposed to paraquat
}

\author{
YONG-SIK KIM $^{1 *}$, HANA JUNG ${ }^{1 *}$, HYO-WOOK GIL ${ }^{2}$, SAE-YONG HONG $^{2}$ and HO-YEON SONG ${ }^{1}$ \\ ${ }^{1}$ Department of Microbiology, College of Medicine, Soon Chun Hyang University, Cheonan, Chungnam 330-090; \\ ${ }^{2}$ Department of Internal Medicine, Soon Chun Hyang Cheonan Hospital, Cheonan, Republic of Korea
}

Received May 30,2012; Accepted August 13, 2012

DOI: $10.3892 / \mathrm{ijmm} .2012 .1143$

\begin{abstract}
Paraquat (PQ) poisoning remains a major public health concern in many countries. Extensive research has focused on finding early diagnostic biomarkers of acute PQ poisoning. In order to investigate the characterization of diagnostic biomarkers in PQ poisoning, we utilized proteomic analysis using serum from rats exposed to $P Q$, and we identified 8 differentially expressed proteins from over 500 protein spots. The expression of apolipoprotein E (ApoE), preprohaptoglobin (Pphg), a precursor of haptoglobin (Hp), and complement component 3 (C3) proteins was greatly induced by PQ exposure while the expression of fibrinogen $\gamma$-chain (FGG) and Ac-158 was dramatically reduced. To further investigate the possibility of ApoE, Pphg and FGG as useful diagnostic biomarkers of PQ poisoning, western blot and qRT-PCR analyses were conducted using cell lines as well as rat and human sera. The expression levels of ApoE, Hp and FGG were significantly altered in the presence of PQ in both rat and human serum suggesting that these proteins may be appropriate candidate molecular biomarkers for the early diagnosis of acute PQ intoxication.
\end{abstract}

\section{Introduction}

Paraquat (trade name Gramoxone ${ }^{\circledR}$; 1,1'-dimethyl-4,4'-bipyridinium dichloride; PQ) is a potent herbicide widely used throughout the world (1). PQ intoxication by accidental and intentional ingestion has been a public health concern for several decades due to the severe morbidity and mortality rates in both developing and developed countries. A variety of clinical approaches for treatment of PQ intoxication have

Correspondence to: Professor Ho-Yeon Song, Department of Microbiology, College of Medicine, Soon Chun Hyang University, 366-1 Ssangyong-dong, Cheonan, Chungnam, 330-090, Republic of Korea

E-mail: songmic@sch.ac.kr

\section{${ }^{*}$ Contributed equally}

Key words: paraquat intoxication, proteomics, haptoglobin, apolipoprotein $\mathrm{E}$ been employed, but highly effective treatment methods have yet to be defined.

The mechanism of $P Q$ poisoning is based on excessive generation of reactive oxygen species (ROS). PQ is reduced by NADPH and then conveys electrons to molecular oxygen generating ROS. This can disrupt the balance of intracellular redox cycling and lead to cellular toxicity $(1,2)$. Ingested PQ directly targets the lungs, kidneys, and liver tissues, causing severe primary cellular damage. PQ toxicity can lead to acute lung phenomena including infiltration of the lungs by inflammatory cells, alveolar hemorrhage, increased collagen deposition and sequential development of mortal lung fibrosis $(1,3)$. Clinical features of PQ ingestion depend on the quantity ingested and the time elapsed from ingestion. Upon ingestion, PQ is rapidly absorbed, distributed to various tissues, and within 12-24 h most of the absorbed PQ is secreted through urine $(4,5)$. Within a few days, patients may develop severe lung damage such as fibrosis, the main cause of mortality with PQ toxicity. Prompt diagnosis and prognosis of pathophysiological progression is crucial for survival. There is, however, a lack of effective diagnostic methods for PQ intoxication due to considerable variations between patients such as age, gender, susceptibility, time elapsed from PQ exposure, and amount of PQ ingested.

Proteomic analysis is one of the most widely used techniques for defining functional proteins and crosstalk between proteins and DNA (6-8). Furthermore, proteomics is a powerful research tool for the identification of biomarkers and therapeutic targets for toxicology, pharmacology, cancer biology, and other biomedical research (8). In pulmonaryrelated disease research, proteomics is a highly effective method for identifying diagnostic and prognostic biomarkers using various biomaterials such as cell lines, lung bronchoalveolar lavage fluid (BALF), and tissues from animals $(9,10)$. However, blood samples have not been considered useful for proteomic analysis despite the fact that blood possesses a highly-enriched information source due to the absence of genomic information (11). Recent developments of advanced proteomic techniques have resulted in the recognition of blood as an important source of information for various biomedical studies such as toxicology (8).

In the present study, we employed a conventional proteomics approach using rat serum to investigate and identify diagnostic biomarkers in PQ poisoning. 2D-PAGE and 
MALDI-TOF analysis revealed PQ treatment altered protein expression. The expression of apolipoprotein E (ApoE), complement component 3 (C3), and preprohaptoglobin (Pphg), a precursor of haptoglobin (Hp), were greatly induced while the expression of fibrinogen $\gamma$-chain (FGG) and Ac1-581, a precursor of fibrinogen $\beta$-chain (FGB), were reduced. Furthermore, alteration of the protein expression of ApoE, Hp and FGG was confirmed in PQ-exposed cell lines and sera from patients. Therefore, our data suggest that Apo E, Hp and FGG may beneficial diagnostic biomarkers for the early detection of acute PQ intoxication.

\section{Materials and methods}

Chemicals and reagents. Paraquat dichloride (1,1'-dimethyl4,4'-bipyridinium dichloride) was purchased from Sigma-Aldrich (St. Louis, MO, USA). The stock solution of PQ was made using distilled water. Stock solutions were then diluted in 1xPBS to the desired concentrations prior to treatment of animals. Stock solutions were diluted in cell culture media for the treatment of cells.

Cell culture. Macrophage, Raw264.7, and lung adenocarcinoma, A549, cell lines were obtained from the Korean Cell Line Bank (Seoul, Korea) and maintained in DMEM and DMEM/HF-12 medium, respectively, supplemented with $10 \%$ $(\mathrm{v} / \mathrm{v})$ heat-inactivated FBS and $1 \%(\mathrm{v} / \mathrm{v})$ antibiotic/antimycotic cocktail $(100 \mathrm{U} / \mathrm{ml}$ penicillin, $100 \mu \mathrm{g} / \mathrm{ml}$ streptomycin, and $0.25 \mu \mathrm{g} / \mathrm{ml}$ amphotericin B; Invitrogen, Carlsbad, CA) at $37^{\circ} \mathrm{C}$ under a humidified atmosphere containing $5 \% \mathrm{CO}_{2}$.

Animals. Six five-week-old male CD(SD)IGS rats were purchased from Orient Bio, Inc. (Seongnam, Korea) and acclimated for 7 days prior to the experiment. Rats were housed with free access to standard rodent food in compliance with the standards set forth by Soon Chun Hyang's Institutional Animal Care and Use Committee.

Ethics statement. Serum samples from patients intixicated with PQ were obtained at the department of internal medicine, Soon Chun Hyang University Cheonan Hospital, and the use of patient serum in this study was approved by Soon Chun Hyang University Cheonan Hospital's institutional review board (IRB approval no. 2011-85).

Proteomics analysis. Serum samples were collected from rats following the guidelines for care and use of laboratory animals approved by Soon Chun Hyang's Institutional Animal Care and Use Committee. Untreated normal blood was initially collected one day prior to PQ treatment (noted as -1 in the text and figures) from the rat tail vein. Blood samples from the tail vein were then collected at $6 \mathrm{~h}$ following intraperitoneal injection of PQ (noted as 1). At Day 3, a final blood collection was performed. Serum samples were immediately collected via $1,500 \mathrm{x}$ g centrifugation at room temperature and samples were stored at $4^{\circ} \mathrm{C}$.

For 2D-PAGE separation, $200 \mu \mathrm{g}$ of serum was used for isoelectric focusing (IEF) using a multiphor II electrophoresis kit following the manufacturer's instructions. For 2D-PAGE, strips were equilibrated in equilibration buffer $(50 \mathrm{mM}$ Tris-
Cl, pH 6.8 containing $6 \mathrm{M}$ urea, 2\% SDS and 30\% glycerol) for $10 \mathrm{~min}$, and the equilibrated strips were inserted and run on $20 \times 24 \mathrm{~cm}$ (10-16\% gradient) SDS-PAGE gels. 2D gels were then stained with modified silver staining according to Oakley et al (12) for image analysis. Quantitative analysis of visualized images was executed using PDQuest (version 7.0; Bio-Rad, Hercules, CA, USA) software following the manufacturer's instructions. We selected the spots with $>2$-fold alteration, normalized by total valid spot intensity. In order to characterize the selected spots, the in-gel digestion was performed using porcine trypsin (Promega; Madison, WI, USA) with modified methods as previously described (13). Digested gel pieces were sequentially washed with $50 \%$ acetonitrile to remove chemicals and dye, and were then dehydrated. Spots were rehydrated with trypsin $(8-10 \mathrm{ng} / \mu 1$ in $50 \mathrm{mM}$ ammonium bicarbonate, $\mathrm{pH} 8.7)$, and incubated for $8-10 \mathrm{~h}$ at $37^{\circ} \mathrm{C}$.

To identify proteins, MALDI-TOF/TOF analysis was conducted. In brief, each spot was verified and analyzed using the TOF/TOF ${ }^{\mathrm{TM}}$ ion optics installed in Applied Biosystems 4700 proteomics analyzer (Applied Biosystems; Carlsbad, CA, USA). Both MS and MS/MS data were acquired with an Nd:YAG laser with $200 \mathrm{~Hz}$ repetition rate, and up to 4,000 shots were accumulated for each spectrum. Operation MS/MS mode was $2 \mathrm{keV}$ collision energy. Air was used as the collision gas with nominally single collision conditions. For these analyses, a resolution of 100 was applied although the prec ursor resolution selection was 200. Both MS and MS/MS data were acquired using the default instrument calibration without applying internal or external calibration. Sequence tag searches were performed with the program MASCOT (http:// www.matrixscience.com).

Quantitative RT-PCR ( $q R T-P C R$ ). Cells were treated with $166 \mathrm{nM}$ PQ for $24 \mathrm{~h}$ and harvested. Total-RNA was purified and converted to cDNA using a first strand cDNA synthesis kit (Intron; Daejeon, Korea). qRT-PCR was performed with human ApoE primer set (forward, 5'-GTGGAGCAAGCGG TGGAGAC-3' and reverse, 5'-GAGCTGAGCAGCTCCTCC TG-3'). As an internal expression control, a GAPDH primer set (forward, 5'-TCCCATCACCATCTTCCA-3' and reverse, 5'-CA TCACGCCACAGTTTCC-3') was used. Amplicon sizes were 158 and $380 \mathrm{bp}$, respectively.

Western blot analysis. In order to measure the Hp and ApoE protein expression from rat and human patient serum, serum albumin was depleted using an albumin depletion kit (Millipore; Billerica, MA, USA) according to the manufacturer's instructions. In brief, $25 \mu 1$ of serum was pre-diluted with $275 \mu 1$ of 1 XPBS and diluted serum was incubated with pre-washed albumin magnetic beads for $6 \mathrm{~h}$ at room temperature. Following incubation, supernatant was isolated and collected for further experiments. Serum protein concentration was measured using the Pierce BCA protein assay kit (Thermo Scientific; Rockford, IL, USA) and mixed with 2XSDS-PAGE sample buffer (Sigma-Aldrich). Subsequently, $35 \mu \mathrm{g}$ serum samples were separated on gradient SDS-PAGE gel and transferred to PVDF membranes. Although we measured the concentration of proteins, gels were stained with the MemCode ${ }^{\mathrm{TM}}$ Reversible Protein stain kit (Thermo Scientific) to check the concentra- 
Table I. The characterized proteins from PQ-exposed rat serum using MALDI-TOF analysis.

\begin{tabular}{|c|c|c|c|c|c|c|c|}
\hline $\begin{array}{l}\text { Spot } \\
\text { no. }\end{array}$ & Sample & GeneBank & $\begin{array}{l}\text { MASCOT search } \\
\text { results file } \\
\text { name }(\mathrm{ms} / \mathrm{ms}) \mathrm{c}\end{array}$ & $\begin{array}{l}\text { Identified } \\
\text { proteins }\end{array}$ & Score & $\operatorname{Cov}(\%)$ & $\begin{array}{c}\text { Changed } \\
\text { pattern }\end{array}$ \\
\hline 1 & 104 & gil37805241 & 104 msms.htm & Apolipoprotein E & 236 & 45 & $\mathrm{Up}$ \\
\hline 2 & 1305 & gil61098186 & 1305 msms.htm & Fibrinogen $\gamma$-chain & 233 & 18 & Down \\
\hline 3 & 1306 & gil61098186 & 1306 msms.htm & Fibrinogen $\gamma$-chain & 121 & 20 & Down \\
\hline 4 & 4103 & gil116597 & 4103 msms.htm & Complement C3 & 121 & 2 & $\mathrm{Up}$ \\
\hline 5 & 4104 & gil116597 & 4104 msms.htm & Complement C3 & 205 & 4 & $\mathrm{Up}$ \\
\hline 6 & 8406 & gil32527707 & 8406 msms.htm & $\begin{array}{l}\text { Ac1-581 } \\
\text { (Fibrinogen } \beta \text {-chain } \\
\text { precursor) }\end{array}$ & 182 & 45 & Down \\
\hline 7 & 1103 & gil204657 & 1103 msms.htm & Preprohaptoglobin & 44 & 10 & Up \\
\hline 8 & 4105 & gil204657 & 4105 msms.htm & Preprohaptoglobin & 67 & 16 & $\mathrm{Up}$ \\
\hline
\end{tabular}



Figure 1. Schematic experimental design. Rats were acclimated for 7 days. Blood samples were collected at the indicated days. White arrow indicates the intraperitoneal injection of $30 \mathrm{mg} / \mathrm{kg}(166 \mathrm{nM}) \mathrm{PQ}$ at Day 1. Gray arrows indicate blood collection at Day $-1,1$ and 3. B indicates blood collection day.

tion of serum proteins after gel running on the SDS-PAGE (Fig. 2), and by transfering to PVDF membranes for antibody incubation. Anti-Hp and anti-ApoE antibodies were diluted at $1: 1,000$ and 1:5,000, respectively.

\section{Results}

Differentially expressed proteins in serum from rats exposed to $P Q$. It is generally difficult to obtain normal serum (as a control) from PQ-intoxicated patients since patients are hospitalized after PQ ingestion, be it intentional or accidental ingestion. Therefore, we mimicked the conditions of PQ poisoning using a rat animal model. Six rats were acclimated at least 7 days prior to the experiments. Normal serum was then collected from the tail following sedation with ether (Fig. 1). Rats were weighed and their general physical condition were checked. After administering $30 \mathrm{mg} / \mathrm{kg}$ (166 nM) PQ via intraperitoneal injection, body weight was found to be slightly reduced but recovered to normal by Day 3 (data not shown). To determine the alteration of functional protein expression in acute PQ-exposed rats, we performed proteomic analysis using $200 \mu \mathrm{g}$ serum samples at 1 day prior to PQ treatment and 1-3 days post-PQ exposure from 2 individual mice.

Representative 2D-PAGE image showed that $>500$ spots clearly altered their expression (Fig. 2). For further characterization of each spot, 8 spots which showed $>2$-fold alteration were collected and MALDI-TOF MS/MS analysis was performed.

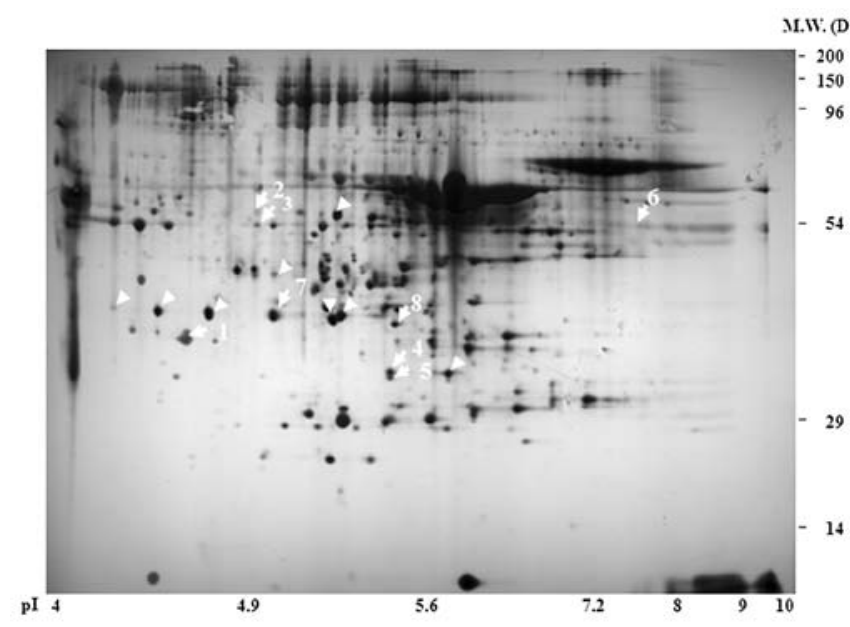

Figure 2. Representative 2DE gel of rat serum proteins which were altered during PQ poisoning. PQ-induced alteration of protein expression was visualized by modified silver staining (12). Arrowheads indicate that the change in protein expression was $>2$-fold when compared with normalized protein expression to the total intensity of valid spots. Numbered arrows indicate proteins identified using MALDI-TOF MS/MS analysis.

We identified 8 proteins of interest including inflammatoryrelated C3, FGG, antioxidant related Pphg, ApoE and Ac1-581 (FGB) via the MASCOT program governed by the National Resource for the Mass Spectrometic Analysis of Biological Macromolecules and the Matrix Science Company (Table I). The MASCOT search program revealed that the mass fingerprint of spot 104 was a $45 \%$ polypeptide match with ApoE protein. The expression of ApoE was significantly upregulated with PQ exposure (Fig. 3A and B). Two individual spots, 4103 and 4104 , were covered with 2 and $4 \% \mathrm{C} 3$, respectively, and the expression of C3 was significantly upregulated in PQ-exposed rat serum in a time-dependent manner (Fig. 3C and D). Of note, 2 spots, 1103 and 4105, were matched with antioxidantrelated Pphg with 10 and $16 \%$ peptide coverage, respectively, and the expression was dramatically induced by PQ treatment (Fig. 3E and F).

The expression of other proteins was found to be downregulated in PQ-exposed rat serum. Spots 1305 and 1306 were 




C
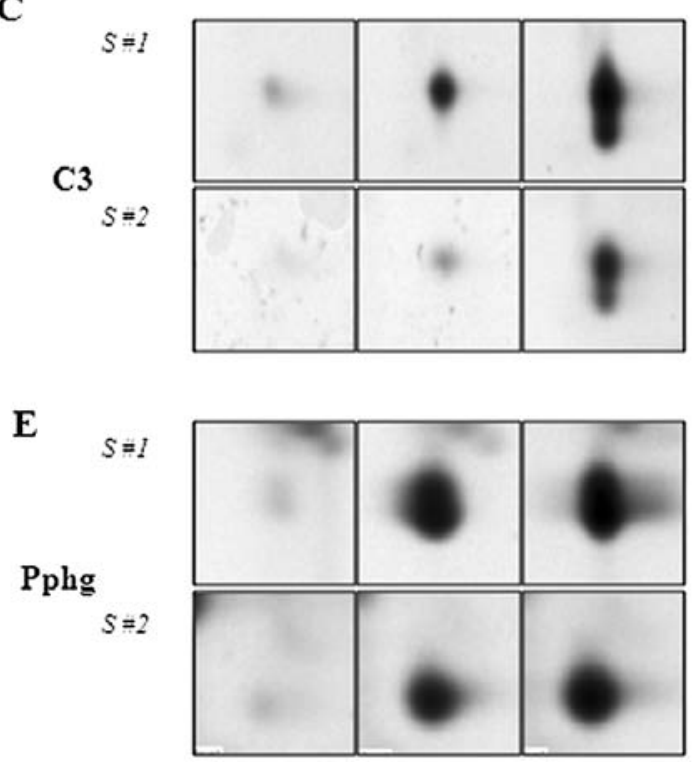

B

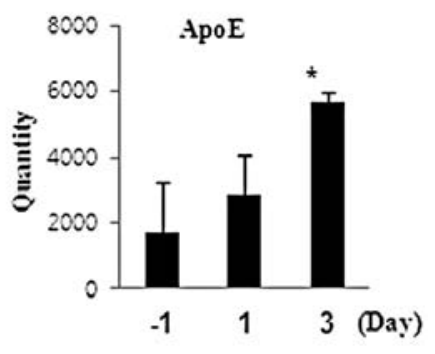

D

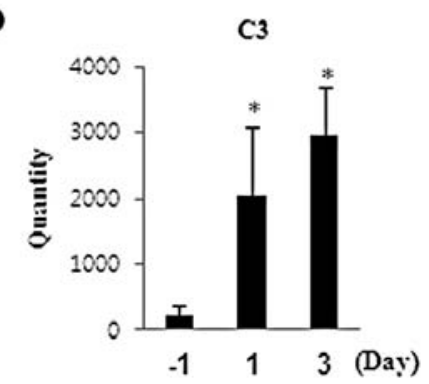

$\mathbf{F}$

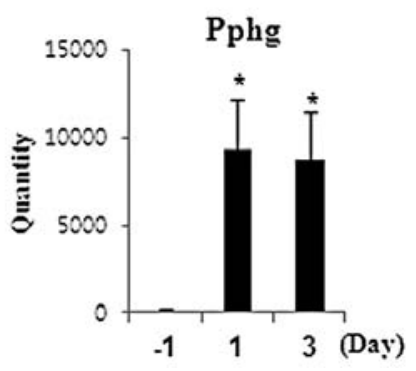

Figure 3. Determination and quantitative analysis of upregulated proteins during PQ poisoning. Upregulated protein expression with PQ treatment was observed. The expression of (A and B) apolipoprotein E, (C and D) complement C3, and (E and F) preprohaptoglobin (Pphg) were shown to be upregulated with PQ treatment. S\#1 and S\#2 indicate 2 different sources of serum in this experiment. Asterisk denotes statistically significant using a paired Student's t-test with p<0.05.

A

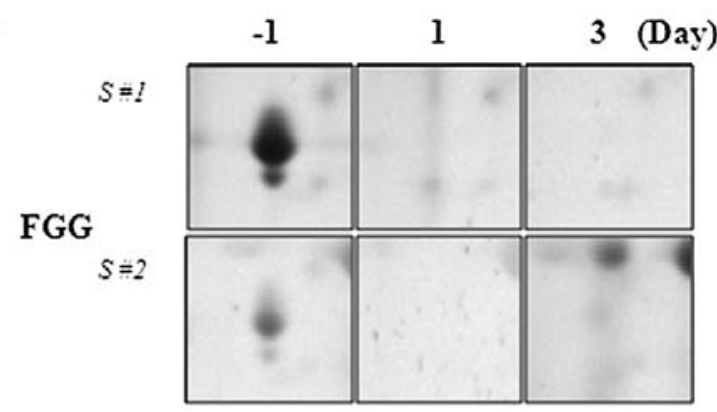

C

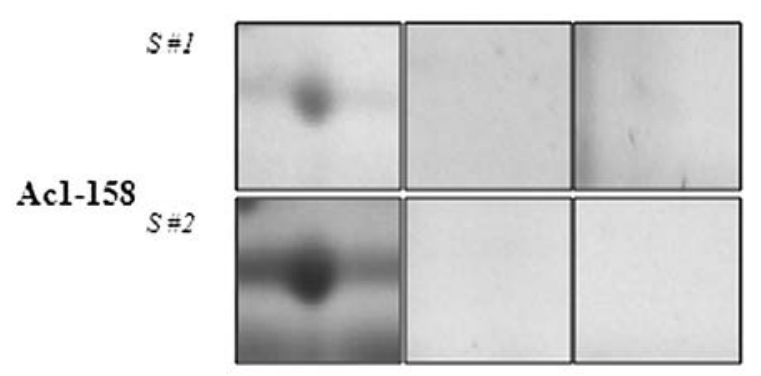

B



D

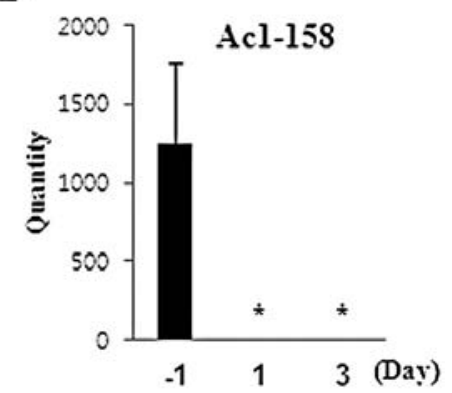

Figure 4. Determination and quantitative analysis of downregulated proteins during PQ poisoning. In order to present data on downregulated protein expression in PQ-exposed serum, (A and B) fibrinogen $\gamma$-chain (FGG), and (C and D) Ac1-158 are shown. S\#1 and S\#2 indicate the 2 different sera used in this experiment. Asterisk denotes statistically significant using a paired Student's t-test with $\mathrm{p}<0.05$. 
A

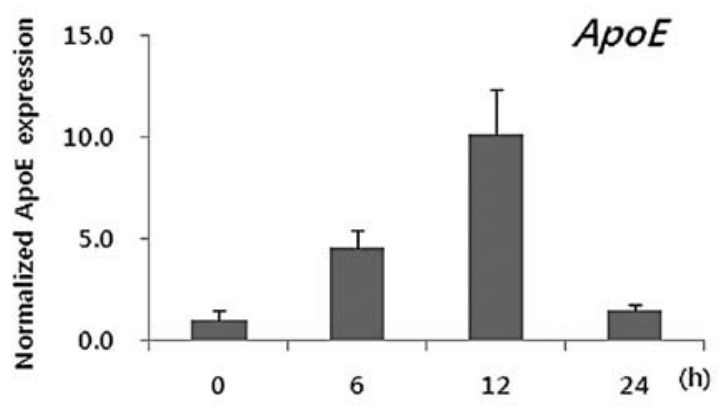

B

Raw264.7

A549

\begin{tabular}{|c|c|c|c|c|c|c|c|c|c|c|}
\hline 0 & 3 & 6 & 12 & 24 & 0 & 3 & 6 & 12 & 24 & (h) \\
\hline chenen & & & s. & Sences? & 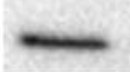 & - & - & & & Hp \\
\hline thes & ment & & $m$ & twon: & $\longrightarrow$ & - & - & & - & ApoE \\
\hline
\end{tabular}

Figure 5. Verification of the altered expression of haptoglobin (Hp) and apolipoprotein E (ApoE). (A) Raw264.7 cell lines were treated with PQ, purified for total-RNA, and converted to cDNA using the first strand cDNA synthesis kit. Quantitative RT-PCR analysis was performed with an ApoE gene specific primer set (described in Materials and methods). (B) Raw264.7 and A549 cells were treated with $166 \mathrm{nM}$ PQ for 0, 3, 6, 12 and 24 h. Proteins were collected and separated on SDS-PAGE gels. Western blot analysis was performed using anti-Hp $(1: 5,000)$ and ApoE antibodies $(1: 1,000)$.
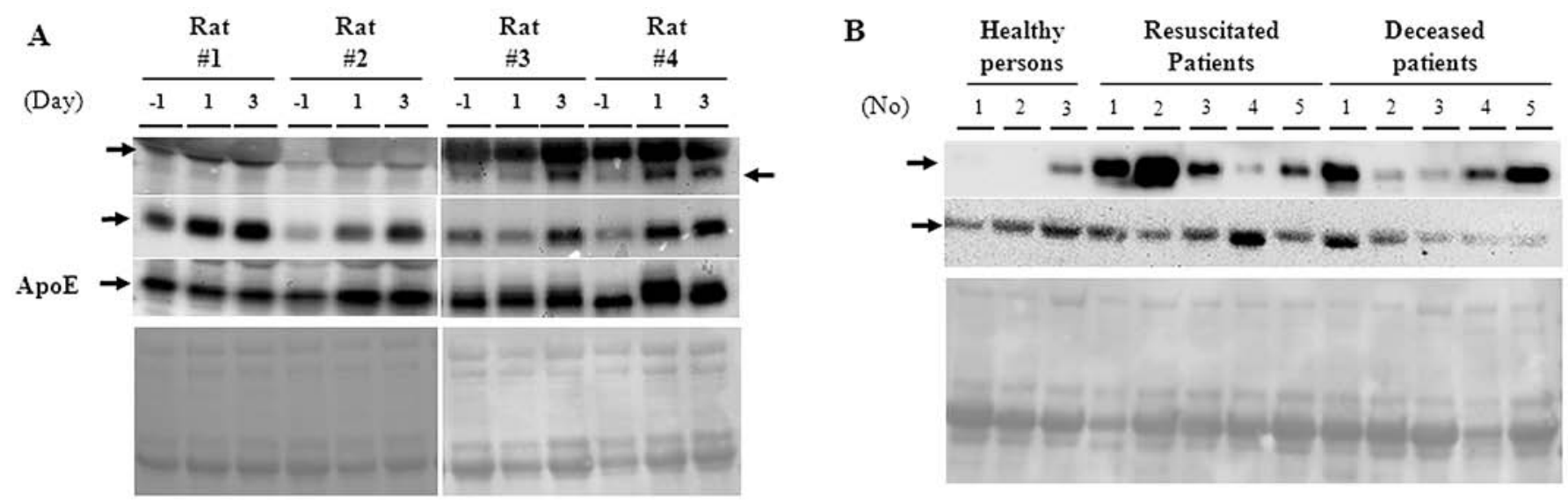

Figure 6. Verification of the altered expression of haptoglobin (Hp) and apolipoprotein E (ApoE). (A and B) After albumin depletion from rat and human serum samples, concentration was measured and $35 \mu \mathrm{g}$ of albumin-depleted serum was mixed with $2 \mathrm{X}$ SDS-PAGE sample buffer and boiled. Each sample was loaded on 4-12\% SDS-PAGE gels and transferred to PVDF membranes. As loading controls, SDS-PAGE gels were stained with the MemCode ${ }^{\mathrm{TM}}$ Reversible protein stain kit. The blots were incubated with anti-Hp antibody $(1: 5,000)$ and anti-ApoE antibody $(1: 1,000)$ overnight at $4^{\circ} \mathrm{C}$. Secondary anti-rabbit antibody $(1: 10,000)$ for anti-Hp and anti-ApoE were treated for $2 \mathrm{~h}$ at room temperature. Detection of FGG was achieved via treatment with anti-FGG antibody $(1: 100$; Santa Cruz Biotechnology, Inc.) and secondary treatment with anti-rabbit IgG antibody (1:10,000). The reactions were visualized using a BioRad Gel Doc system with ECL treatment.

covered with 18 and $20 \%$ FGG polypeptide, respectively, and the expression of FGG was drastically downregulated by PQ (Fig. 4A and B). Markedly, Ac1-581 (spot 8406) which is the preproprotein precursor of FGB was identified, with $45 \%$ coverage, using the MASCOT search engine, and found to be downregulated by PQ exposure in a time-dependent manner (Fig. 4C and D).

Verification of the expression of APOE, Hp and FGG in $P Q$-exposed cell lines, rat and patient sera. To verify the change in mRNA and protein expression with PQ treatment, in vitro analysis was performed. In order to measure the change in mRNA expression, the Raw264.7 cell line was treated with $166 \mathrm{nM}$ PQ for the indicated times $(0,1$ and 3 days). Total-RNA was then collected and cDNA was synthesized using $1 \mu \mathrm{g}$ of total-RNA. qRT-PCR was performed with the ApoE gene specific primer set (described in Materials and methods). ApoE mRNA was drastically upregulated at 6 and $12 \mathrm{~h}$ with PQ treatment (Fig. 5A). However, the expression was downregulated at $24 \mathrm{~h}$. In addition, we investigated the 
expression of the $\mathrm{Hp}$ and ApoE proteins in the PQ-treated Raw264.7 and A549 cell lines (Fig. 5B). As we expected, the expression of the $\mathrm{Hp}$ and ApoE proteins was upregulated at 6 and $12 \mathrm{~h}$ in Raw264.7 cells and A549 cells after PQ treatment, respectively (Fig. 5B).

In order to investigate any possible implication for clinical relevance, we measured the expression of both ApoE and $\mathrm{Hp}$ proteins in rat and human serum. In Fig. $6 \mathrm{~A}$, the Hp- $\alpha,-\beta$ proteins, and ApoE were dramatically induced by PQ treatment in a time-dependent manner. Hp- $\beta$ proteins were also prominently induced in both resuscitated and deceased PQ patients compared to healthy individuals (Fig. 6B). By contrast, expression of FGG was downregulated in PQ patients.

\section{Discussion}

Due to the high rate of mortality with poisoning, prompt prognosis and diagnosis of acute PQ poisoning is an absolute requirement for survival. Serum uric acid and the acutephase response gene pentraxin 3 (PTX3) were recently characterized and highlighted as putative biomarkers for $\mathrm{PQ}$ poisoning in human serum $(14,15)$. However, the protocols for diagnosis, prognosis, and treatment of $\mathrm{PQ}$-poisoned patients are limited. These limitations led us to investigate the existence of potentially more effective diagnostic biomarkers from PQ-treated rat serum using conventional proteomics analysis. Although there was concern of protein spots being masked by high-abundance serum proteins such as albumin, immunoglobulin, and complement on 2D-PAGE images, we were able to perform proteomic analysis with total serum to identify putative biomarkers. More than 500 altered protein spots were gained, and we identified 8 differentially expressed proteins. The expression of ApoE, C3 and Pphg, a precursor of haptoglobin (Hp), were induced by PQ treatment while the FGG and Ac1-581 (FGB) proteins were downregulated. The altered protein expressions were further verified by qRT-PCR and western blot analysis. In addition, we detected the induction of ApoE and $H p$ mRNA expression in the A549 lung carcinoma cell line. This data suggested that these proteins may be beneficial candidate markers for the diagnosis of acute PQ poisoning.

Haptoglobin is an acute-phase response glycoprotein and it plays an antioxidative role due to its binding activity to hemoglobin (16). Haptoglobin is also a good diagnostic marker of lung cancer and is involved in angiogenesis and cell migration $(17,18)$. Li et al $(20)$ also demonstrated that $\mathrm{Hp}$ is upregulated in acute lung damage after pulmonary embolism (19). A human study demonstrated that serum Hp concentration was significantly increased from approximately 824.37 to $2063 \mathrm{mg} / \mathrm{l}$ in patients with pulmonary embolism and deep vein thrombosis (PE/DVT) (20). Based on these results, studies have suggested that increasing concentrations of serum Hp may attenuate lung injury that occurs following PE. Karthik et al (21) demonstrated that the expression of an Hp precursor, Pphg, was greatly altered in the alloxan-induced diabetes model. In addition, the expression of Pphg and $\mathrm{Hp}$ in patient urine was dramatically increased in the presence of passive Heymann nephritis and suggested that Pphg and $\mathrm{Hp}$ are appropriate candidates for therapeutic targeting and potentially novel biomarkers in membranous nephropathy (MN) and high altitude pulmonary edema $(22,23)$. We identified Pphg via proteomic analysis and observed that its expression was dramatically induced in PQ-treated rat serum. Prior to treatment with PQ, Pphg expression was almost undetectable but expression was significantly induced at Days 1 and 3 post-PQ administration. Furthermore, the expression of $\mathrm{Hp}-\alpha$ and $\mathrm{Hp}-\beta$ was detected in PQ-treated rat and human serum. In accordance with previous studies, we speculated that the induction of Pphg and Hp expression was a response to PQ treatment, therefore, Pphg and Hp serum levels may be effective diagnostic biomarkers for $\mathrm{PQ}$ poisoning.

Accumulating evidence from animal studies has revealed that $\mathrm{ApoE}$ is a key protein in atherosclerosis via its role in inflammation, control of cholesterol, and blood pressure (24). A previous study demonstrated that $\mathrm{Hp}$ can interact with ApoE in a mechanism by which inflammation affects atherosclerotic progression (25). Cigliano et al (25) suggested that the interaction between Hp and ApoE represents a novel link between the acute phase of inflammation and ApoE function. In addition, the ApoE knockout mice, a lack of the antioxidant enzyme, and glutathione peroxidase-1 (GPx1), accelerate diabetesassociated atherosclerosis through induction of inflammation and fibrosis (19). However, Cuthbert et al (26) demonstrated that the level of expression of the major protein component of high-density lipoprotein (HDL), apolipoprotein A-I (ApoA-I) but not ApoB and E, is regulated by PQ exposure in the HepG2 cell line. In this study, we observed that the expression of the ApoE protein was dramatically induced by PQ treatment. Based on our observation, we speculated that early acute PQ exposure may induce ApoE which leads to the regulation of the acute inflammatory response and antioxidant-related genes. The dynamic expression among the Apo family of proteins requires further investigation in order to better understand the regulatory network between PQ and the early immune response.

Although fibrinogen $\gamma$-chain (FGG) is known to be a cofactor in blood clots, FGG and fibrinogen $\beta$-chain (FGB) are also known as biomarkers for the detection of occurrence and progression of coronary artery disease (CAD) in $A p o E^{-1-}$ mice (27). FGG is also involved in aging through the regulation of oxidative stress and inflammation (28). In this study, FGG was dramatically downregulated by PQ exposure. To date, there has been no data regarding the role of FGG in $P Q$ intoxication. Therefore, we speculated that the antagonistic expression between ApoE and FGG is in direct response to acute $\mathrm{PQ}$ poisoning.

It has been well established that PQ induces ROS formation, and, consequently, development of acute lung injury such as lung fibrosis via induction of the NF- $\mathrm{BB}$ regulated proinflammatory pathway (1). Complements have been involved in early immune reaction but complement component 3 (C3) levels in serum were not altered with long-term PQ exposure in Balb/c mice (29). Sun et al (30) demonstrated that blocking the complement pathway ameliorates acute lung injury (ALI) induced by PQ treatment. The treatment of complement $\mathrm{C} 3$ inhibitor, CR2-Crry, has been shown to be particularly effective in reducing PQ-induced inflammation, pathology, and mortality (30). Data suggest that C3 may play an important role in acute, early inflammatory reactions induced by $\mathrm{PQ}$. In the present study, $\mathrm{PQ}$ induced expression of $\mathrm{C} 3$ which was shown to activate the acute pro-inflammatory response and 
subsequent inflammation. Therefore, we propose that the detection of $\mathrm{C} 3$ expression in serum is a beneficial diagnostic tool for PQ-induced acute lung inflammation.

In conclusion, based on our proteomics profiling data we suggest that Hp, ApoE, C3 and FGG in PQ-exposed serum might be appropriate diagnostic biomarkers for the early detection and diagnosis of acute PQ poisoning.

\section{Acknowledgements}

This study was partially supported by a grant from the Rural Development Administration, Republic of Korea (project no. PJ008246).

\section{References}

1. Gawarammana IB and Buckley NA: Medical management of paraquat ingestion. Br J Clin Pharmacol 72: 745-757, 2011.

2. Ahmad I, Kumar A, Shukla S, Prasad Pandey H and Singh C: The involvement of nitric oxide in maneb- and paraquat-induced oxidative stress in rat polymorphonuclear leukocytes. Free Radic Res 42: 849-862, 2008.

3. Suntres ZE: Role of antioxidants in paraquat toxicity. Toxicology 180: 65-77, 2002.

4. Houze P, Baud FJ, Mouy R, Bismuth C, Bourdon R and Scherrmann JM: Toxicokinetics of paraquat in humans. Hum Exp Toxicol 9: 5-12, 1990.

5. Pond SM, Rivory LP, Hampson EC and Roberts MS: Kinetics of toxic doses of paraquat and the effects of hemoperfusion in the dog. J Toxicol Clin Toxicol 31: 229-246, 1993.

6. Indovina P, Marcelli E, Maranta P and Tarro G: Lung cancer proteomics: recent advances in biomarker discovery. Int $\mathrm{J}$ Proteomics 2011: 726869, 2011.

7. Lau AT and Chiu JF: Biomarkers of lung-related diseases: current knowledge by proteomic approaches. J Cell Physiol 221: 535-543, 2009.

8. Plymoth A and Hainaut P: Proteomics beyond proteomics: toward clinical applications. Curr Opin Oncol 23: 77-82, 2011.

9. Govender P, Dunn MJ and Donnelly SC: Proteomics and the lung: Analysis of bronchoalveolar lavage fluid. Proteomics Clin Appl 3: 1044-1051, 2009.

10. Okamoto T, Miyazaki Y, Shirahama R, Tamaoka M and Inase N: Proteome analysis of bronchoalveolar lavage fluid in chronic hypersensitivity pneumonitis. Allergol Int 61: 83-92, 2012.

11. Issaq HJ, Xiao Z and Veenstra TD: Serum and plasma proteomics. Chem Rev 107: 3601-3620, 2007.

12. Oakley BR, Kirsch DR and Morris NR: A simplified ultrasensitive silver stain for detecting proteins in polyacrylamide gels Anal Biochem 105: 361-363, 1980 .

13. Shevchenko A, Wilm M, Vorm O and Mann M: Mass spectrometric sequencing of proteins silver-stained polyacrylamide gels. Anal Chem 68: 850-858, 1996.

14. Kim JH, Gil HW, Yang JO, Lee EY and Hong SY: Serum uric acid level as a marker for mortality and acute kidney injury in patients with acute paraquat intoxication. Nephrol Dial Transplant 26: $1846-1852,2011$.
15. Yeo CD, Kim JW, Kim YO, Yoon SA, Kim KH and Kim YS: The role of pentraxin-3 as a prognostic biomarker in paraquat poisoning. Toxicol Lett 212: 157-160, 2011.

16. Yang F, Ghio AJ, Herbert DC, Weaker FJ, Walter CA and Coalson JJ: Pulmonary expression of the human haptoglobin gene. Am J Respir Cell Mol Biol 23: 277-282, 2000.

17. Salomonsson E, Thijssen VL, Griffioen AW, Nilsson UJ and Leffler $\mathrm{H}$ : The anti-angiogenic peptide anginex greatly enhances galectin-1 binding affinity for glycoproteins. J Biol Chem 286: 13801-13804, 2011.

18. Tsai HY, Boonyapranai K, Sriyam S, et al: Glycoproteomics analysis to identify a glycoform on haptoglobin associated with lung cancer. Proteomics 11: 2162-2170, 2011.

19. Lewis P, Stefanovic N, Pete J, et al: Lack of the antioxidant enzyme glutathione peroxidase-1 accelerates atherosclerosis in diabetic apolipoprotein E-deficient mice. Circulation 115: 21782187, 2007.

20. Li SQ, Yun J, Xue FB, et al: Comparative proteome analysis of serum from acute pulmonary embolism rat model for biomarker discovery. J Proteome Res 6: 150-159, 2007.

21. Karthik D, Ilavenil S, Kaleeswaran B, Sunil S and Ravikumar S: Proteomic analysis of plasma proteins in diabetic rats by $2 \mathrm{D}$ electrophoresis and MALDI-TOF-MS. Appl Biochem Biotechnol 166: 1507-1519, 2012.

22. Ahmad Y, Shukla D, Garg I, et al: Identification of haptoglobin and apolipoprotein A-I as biomarkers for high altitude pulmonary edema. Funct Integr Genomics 11: 407-417, 2011.

23. Ngai HH, Sit WH, Jiang PP, Thongboonkerd V and Wan JM: Markedly increased urinary preprohaptoglobin and haptoglobin in passive Heymann nephritis: a differential proteomics approach. J Proteome Res 6: 3313-3320, 2007.

24. Imaizumi K: Diet and atherosclerosis in apolipoprotein E-deficient mice. Biosci Biotechnol Biochem 75: 1023-1035, 2011.

25. Cigliano L, Pugliese CR, Spagnuolo MS, Palumbo R and Abrescia P: Haptoglobin binds the antiatherogenic protein apolipoprotein E - impairment of apolipoprotein E stimulation of both lecithin: cholesterol acyltransferase activity and cholesterol uptake by hepatocytes. FEBS J 276: 6158-6171, 2009.

26. Cuthbert $C$, Wang Z, Zhang $X$ and Tam SP: Regulation of human apolipoprotein A-I gene expression by gramoxone. J Biol Chem 272: 14954-14960, 1997.

27. Jing L, Parker CE, Seo D, et al: Discovery of biomarker candidates for coronary artery disease from an APOE-knock out mouse model using iTRAQ-based multiplex quantitative proteomics. Proteomics 11: 2763-2776, 2011.

28. Santos-Gonzalez M, Gomez Diaz C, Navas P and Villalba JM: Modifications of plasma proteome in long-lived rats fed on a coenzyme Q10-supplemented diet. Exp Gerontol 42: 798-806, 2007.

29. Riahi B, Rafatpanah H, Mahmoudi M, et al: Evaluation of suppressive effects of paraquat on innate immunity in Balb/c mice. J Immunotoxicol 8: 39-45, 2011.

30. Sun S, Wang H, Zhao G, et al: Complement inhibition alleviates paraquat-induced acute lung injury. Am J Respir Cell Mol Biol 45: 834-842, 2011. 